\title{
DESARROLLO DEL CAMPO LABORAL EN EL TRABAJO SOCIAL DE COSTA RICA DURANTE EL SIGLO XX (1942-2000)
}

\section{DEVELOPMENT OF LABOR ACTIVITY IN THE SOCIAL WORK IN COSTA RICA DURING XX CENTURY (1942-2000)}

\author{
Freddy Esquivel Corella*
}

RESUMEN

El presente artículo es el resultado de una investigación bibliográfica y documental sobre la gestación profesional del Trabajo Social en la Costa Rica del siglo xx. Se realiza un estudio sobre las luchas alcanzadas en la academia, el campo profesional e institucional y los movimientos de articulación, tanto para el reconocimiento legal como de sus atribuciones particulares.

PALABRAS CLAVE: COSTA RICA * TRABAJO SOCIAL * EMPLEO * ESTADO

ABSTRACT

This article is the result of a literature study on the establishment of Social Work professional in Costa Rica during the twentieth century. The axis of the paper is the analysis of the achievements and limitations of this profession in the academia, employment and its institutional meaning.

KEYWORDS: COSTA RICA * SOCIAL WORK * EMPLOYMENT * STATE

Escuela de Trabajo Social de la Universidad de Costa Rica (UCR).

freddy.esquivel@ucr.ac.cr. 


\section{INTRODUCCIÓN}

El Trabajo Social se constituye en una profesión que encuentra su origen en las transformaciones más amplias de la sociedad occidental europea, con una extensa gama de expresiones que la institucionalizaron en diversas naciones (Manrique, 1982).

El agitado contexto de la vida humana, posterior a la Segunda Guerra Mundial, colocó un escenario internacional bajo la hegemonía de los Estados Unidos de Norteamérica (eeuu), donde esta profesión fue intensamente instrumentalizada para coadyuvar (con serias tensiones) a legitimar nuevas rutas de configuración del orden capitalista, por tanto, es posible asentir que esta categoría mantiene una presencia reconocida en la mayoría de los países (Martinelli, 1997).

De manera ilustrativa, cabe ejemplificar con algunos de los resultados de los censos mundiales de enseñanza del Trabajo Social de los años 1950, 2010 y 2012: a mitad del siglo xx se estimaba un total de 373 instituciones en 43 países (Universidad de Costa Rica-ucR, 1954) y sesenta años después se registraron 2000 programas de titulación en educación terciaria en dicho campo.

En el 2012, el dato de centros se localiza en 2110 en 125 estados, donde la consolidación de esos nuevos números se incrementa en Asia, Europa del Este y África (China brindó un el elevado crecimiento sumando 200 programas universitarios en esta especialidad). A su vez, los niveles de titulación en maestría, doctorado, posdoctorado y especialización que se están recolectando, dan cuenta de una cualificación sobresaliente (International Association Schools of Social Work-IAssw, 2013).

Por su lado, la Federación Internacional de Trabajadores(as) Sociales (FITs), fundada en 1928 (París, Francia) articula más de 90 organizaciones de ese gremio alrededor del mundo, contabilizando 750000 personas inscritas. Dicho órgano se ha constituido como consultor de ciertas políticas que desarrolla el Consejo Económico Social de la Organización de las Naciones Unidas (onu), así como, El Fondo de las Naciones Unidas para la infancia (uniceF por sus siglos en inglés), sumado a ello mantiene estrechos lazos con la Organización Mundial de la Salud (омs) y en especial con la Oficina del Alto Comisionado para Refugiados y su similar en Derechos Humanos en la onu (International Federation of Social Workers-IFsw, 2013).

En América Latina, la república donde al parecer existen más unidades formativas en Trabajo Social (Servi o Social) es Brasil, la cual concentraba para el 2010, un número superior a 300 (Conselho Federal de Serviço Social-cress, 2010); una segunda nación, bastante distante en geografía y número es México, con un dato relativo de 47 escuelas de carácter universitario; seguida por Colombia con alrededor de 29 centros de enseñanza (Acosta, Bohórquez, Duarte, González y Rodríguez, 2012). Lo anterior arroja un estimado de más de 400 espacios formativos en el continente, según datos preliminares brindados por la Escuela de Trabajo Social de la Universidad de Costa Rica (2013).

Los números antes acotados son estimaciones muy imprecisas, por cuestiones de subregistro y/o desinformación; aún con esas limitaciones, en América Latina es posible asentir que el dígito de escuelas y graduadas (os) se ha incrementado considerablemente, por ejemplo, se señalaba la existencia de 64 centros de enseñanza en 1954, 183 para 1970 y 210 en 1975 (Castillo, 1980). Por su parte, Alayón (1980) afirmó que para 1978 se ubicaron 236 y hay datos de unas 260 instituciones con esos rasgos para 1980 (Lima, 1980).

Tal proliferación de órganos de titulación en Trabajo Social, vino acompañada históricamente de un aumento significativo de agentes en esa área; aunado a particulares formas de reproducción laboral que se pueden explicar a partir de sus demandas de contratación y remuneración, requerimientos de organización y legitimación social, agendas de control, sanción $y$ supervisión del ejercicio de las facultades que brinda el título académico, así como, sus diversas concatenaciones con los nichos ocupacionales institucionales, principalmente estatales.

En varias partes del mundo y en América Latina en específico, se movilizaron recursos profesionales para consolidar marcos de reconocimiento público estatal y así direccionar, proteger $y$ fortalecer el afianzamiento de dicha 
categoría en los contextos donde se arraigaba (Netto, 1992).

En Costa Rica, las condiciones históricas en que se reprodujo el Trabajo Social en el entramado institucional, han sido poco abordadas de manera panorámica, prevaleciendo lecturas parceladas o bien detenidas en lapsos de tiempo bastante reducidos.

El presente artículo tiene por objetivo hilvanar una serie de investigaciones $y$ fuentes documentales que permitan una asimilación más totalizante de algunas de sus diferentes tensiones y fuerzas socio-históricas que han matizado su reproducción laboral; lo anterior con el fin de lograr algunas conclusiones aproximativas en la materia para la contemporaneidad.

Este documento ha sido elaborado a partir de la investigación documental, bibliográfica $y$ de legislación que se asocia al tema, con fuentes brindadas por diferentes instancias, centros de documentación y bibliotecas privadas donde se conservan algunos textos relacionados con el desarrollo del Trabajo Social.

Los criterios de búsqueda y análisis se centraron en aquellas elaboraciones que daban algunos rasgos, señalamientos y evidencias del entramado de esta profesión con la plataforma institucional costarricense, así como de su reproducción académica y gremial.

\section{CONTEXTO DE CONSOLIDACIÓN DE LAS} PROFESIONES EN COSTA RICA COMO MARCO PARA EL TRABAJO SOCIAL

En la sociedad costarricense se encuentran una serie de intervenciones de diferente naturaleza que se trasponían, traslapaban, encadenaban $y$ tomaban cuerpo (amorfamente inclusive) en lo que refiere a la atención de las condiciones de vida de los sectores más precarizados.

Heredadas desde la era liberal, luego de la independencia de España (1821), los requerimientos de acciones $y$ regulaciones sociales eran latentes, prueba de ello fue la Ley de Bases y Garantías de 1841 en el mandato de Braulio Carrillo Colina, como también lo fue la Ley Reglamentaria de la Administración Local de los Departamentos y la Policía de ese mismo estadista (Calderón, 1988).
Las líneas liberales de regulación de la sociedad costarricense enfocadas en el campo de la salud, la educación, el control social, la economía y en alguna medida la vida civil secular, paulatinamente fueron moldeando prácticas, cargos, labores y oficios en esos ámbitos que eran lo posible de alcanzar para un país con vastas limitaciones académicas, así como, dependiente de una economía agrícola de subsistencia (Obregón, 1981).

La formación de profesionales se fue edificando con sustantivas limitaciones tanto en sus insumos académicos, como en razón de las demandas nacionales; se priorizó en tener cuadros en el campo del Derecho, la Filosofía, la Matemática y la Teología para que le dieran soporte a la edificación del Estado-Nación primigenio del siglo xix (González, 2003).

Cabe ilustrar que la primera universidad de este país se funda en 1843 de forma limitada y con fuertes restricciones de accionar presupuestario e intelectual (González, 1991). Sin embargo, fue clausurada en 1888 por una serie de argumentos entre los que desfilaba la demanda de una formación básica, que diera un recurso humano con una visión más técnica, por lo que se consideró necesario tomar ese dinero para fortalecer la educación primaria y secundaria. Léase al respecto un fragmento de su decreto de cierre:

Considerando: 1. Que la Universidad nacional no tiene organizadas las facultades que constituyen la vida propia de esta institución. 2. Que las actuales condiciones del país no son medio suficiente para organizar un centro de investigación puramente científico. 3 . Que los estatutos y demás disposiciones que rigen la Universidad nacional no concuerdan con los progresos de la ciencia ni con los medios de nuestra condición social. 4. Que es indispensable la reforma de esas leyes y la creación de los elementos necesarios para que los estudios superiores puedan desarrollarse en toda su extensión. [...] Por tanto, y en uso de la atribución que le confiere la Carta Fundamental en su artículo 73, 
inciso 21, DECRETA: Art. 1. Mientras las condiciones sociales del país no permitan la creación de una Universidad como elemento corporativo con la organización que a sus funciones corresponden, queda abolida esta institución; $y$ en su reemplazo créanse Escuelas superiores profesionales de Derecho y Notariado, de Ingeniería y de Medicina. Art. 2. Estas escuelas tendrán gobierno propio, $y$ en ellas intervendrá el Poder Ejecutivo, de acuerdo con la Constitución y la Ley fundamental de Instrucción Pública. Art. 3. Destinase exclusivamente al sostenimiento de aquellas escuelas el capital consolidado de la Universidad nacional extinguida y las demás asignaciones del presupuesto general (Congreso constitucional, 1888: 1).

Disuelta la Universidad de Santo Tomás, los colectivos profesionales quedaron bajo la regulación de otras instancias que ejercían una tarea educativa y de control, aunque con un mayor talante corporativista; ejemplo de ello fue el caso de Derecho, Farmacia, Ingeniería, Cirugía Dental y Bellas Artes, incluso para 1923, había más escuelas que las que se dieron en la extinta institución (Tinoco, 1983).

Al parecer, en esos colegios prevalecía la orientación formativa que se había centrado en la obtención de conocimientos para el trabajo profesional cotidiano (De la Cruz, 2003).

El traslado de responsabilidad de la formación a los órganos profesionales, significó a su vez, centralizar en ciertos grupos privilegiados la direccionalidad curricular y el valor sociohistórico de esos gremios por casi medio siglo, por lo que las raíces liberales de esas organizaciones no causaron mayores tensiones a la teleología de las fracciones en el gobierno que las tutelaban.

Otros entes que ampliaron la formación superior fueron la Sociedad Científico Literaria (1872) y la Academia de Ciencias Sociales (1876), donde se dictaban contenidos de Filosofía, Derecho Natural, Historia y Literatura; esta última era dirigida por el Dr. Antonio Zambrana. En 1879, el Dr. Antonio Espinal, funda la
Academia Pestalozziana de Enseñanza Primaria $y$ en 1881, se inaugura la Academia de Jurisprudencia. Por otro lado, se organizó una Escuela Nacional de Agricultura, Artes Mecánicas y Oficios y en 1886, se inaugura la Escuela Normal para la Enseñanza (Quesada, 2005).

Así las cosas, la vida intelectual y de preparación profesional en el país, que adoleció de una universidad hasta 1940 (Universidad de Costa Rica-ucR), se aglutinó en un conjunto de organizaciones gremialistas, entre estas escuelas de diferentes campos.

De tal manera, como se expondrá más adelante, deja de ser extraño que la naturaleza académico-gremial del Servicio Social, arrancara en una Escuela fuera de la ucR (1940), las cuales apenas tenían dos años de diferencia en su data fundacional.

A juicio personal, las condiciones de precariedad de vida en el caso costarricense, fueron exigiendo diversas pautas de acción, aunado a que la llegada del siglo xx, transfiguró profundamente el modo de vida nacional con la consolidación del capitalismo y las agendas de confrontación político-ideológicas, incluso entre las fracciones hegemónicas (Acuña y Molina, 1991).

La necesidad de respuestas cada vez más latentes hacia las precarias condiciones de vida de la mayoría de habitantes de este pequeño país, se particularizó por la presión de la organización trabajadora aglutinada en asociaciones, partidos o sindicatos que forcejaban por alteraciones, tanto en la administración pública y fiscal, como en razón de que la legislación regulara la explotación del trabajo, la seguridad social y prestara atención a ciertos campos como la vivienda popular, la protección a la infancia $y$ la ampliación de la cobertura educativa (remítase a la Constitución Política de Costa Rica, 1949, Capítulo V Derechos y Garantías Sociales).

De tal forma, se sostiene que la "cuestión social"1 impactaría en los gremios existentes

\footnotetext{
1 Entiéndase básicamente como las tensiones y luchas contra las formas de expoliación que padecían un número significativo de personas por medio del trabajo asalariado (consúltese Esquivel, 2012).
} 
de médicos, abogados, enfermeras y educadoras (es), en tanto, el Estado y los gobiernos de turno iban constituyendo algunas directrices para paliar el pauperismo existente $y$ que les implicaba en ciertas actuaciones; junto a ello, requirió darle preparación a nuevos cuadros como el Trabajo Social, la Orientación Educativa, la Psicología e incluso, aunque más adelante, la Sociología y la Antropología (Molina y Palmer, 2003).

Las diferentes profesiones y agentes del Estado fueron entretejiéndose con la "cuestión social", misma que no es un objeto exclusivo del Trabajo Social, aunque es posible localizar determinantes materiales (objetivas) y subjetivas que particularizan sus nexos históricos (Esquivel, 2007).

En el caso de Costa Rica, esas interrelaciones del Trabajo Social con la "cuestión social", son determinadas fuertemente por la vía institucional que el Estado configuró en el marco de las demandas del capitalismo local e internacional, conjugándose sincréticamente con una plataforma profesional especialmente heredada de las orientaciones del Social Work proveniente de los Estados Unidos (Beeche, 1951).

\section{REFERENTES DEL SURGIR ACADÉMICO DEL TRABAJO SOCIAL EN COSTA RICA}

La fundación de las escuelas en este campo profesional adolece de erigirse como una explicación totalizadora del Trabajo Social en las diferentes sociedades; sin embargo, se constituyen como una base determinante para penetrar en el significado socio-histórico de su naturaleza.

Como se señaló anteriormente, la primera escuela de Servicio Social en el país se establece en 1942 y a pocos meses es integrada a la ucR, tal y como se refiere a continuación:

Así, en 1944, la Escuela de Servicio Social fue aceptada por el Consejo Universitario como escuela anexa a la Facultad de Ciencias Económicas y Sociales de la Universidad de Costa Rica. Durante 3 años, la Escuela de Servicio Social conservó el carácter de anexa... En su condición de anexa, no era equiva- lente a las demás escuelas universitarias, pues sus alumnos y profesores no formaban parte de la Asamblea Universitaria, ni sus títulos podían tener el rango y distinción de los impartidos directamente por las escuelas universitarias... Fue así como la Escuela de Servicio Social, que en casi todas las partes del mundo, constituye una escuela de carácter vocacional, vino a constituir en Costa Rica una escuela universitaria, en la que el espíritu académico predomina sobre el vocacional... Para cursar [en 1955] estudios en esta rama de la Escuela de Ciencias Económicas y Sociales es necesario, al igual que en todas las otras, ser Bachiller en Humanidades y para alcanzar el título de Licenciado, es necesario cursar cinco años de estudios en su gran mayoría teóricos, de manera que el graduado de esta rama tiene, en lugar del carácter de "visitador social", que era lo que se proponía en su origen la Escuela de Servicio Social, la condición de "Investigador Social", que se nos ocurre corresponde mejor a su condición académica actual (Yglesias, 1953: 71-72).

Expresamente hasta 1947, dicha unidad pasa a ser parte orgánica de lo que entonces se delimitaría como Ciencias Económicas y Sociales en condición de Departamento de Servicio Social (que en cierto momento, pero sin consolidarse como tal, se registró como Sociología y Servicio Social) otorgándose una Licenciatura en Ciencias Económicas y Sociales con especialización en Servicio Social (ucR, 1948 y 1954).

Para ese momento, el conjunto de profesionales especializados en esta área que se registraban con licenciatura, escaseaban significativamente, en tanto lo que generalmente se realizaban eran cursos asociados al campo del Servicio Social; ante tal situación se integra como vía alternativa la obtención de un diploma de reconocimiento público en el campo, pero sin grado académico.

...para quienes no tengan interés en la más amplia preparación académica $y$ para la investigación que el grado de 
Licenciatura implica, la Facultad ha formulado un Plan de tres años de estudios al final de los cuales otorga un Certificado de Conclusión de Estudios de Servicio Social (ucR, 1955: 65).

Esta titulación se justificaba de la siguiente manera:

Si los gobiernos nacionales se deciden a valorizar debidamente al técnico y admitir que la Administración Pública debe ser realizada bajo el requerimiento de normas científicas, los egresados... vendrán a ser los factores fundamentales del mejoramiento institucional de la República [por tanto podrán] contar con elemento técnicamente apto para prestar con eficiencia, ya en el campo práctico, los servicios sociales que en número cada vez mayor va el Estado imponiéndose como obligación (UCR, 1948: 4).

El nivel académico de Licenciados (as) en Ciencias Económicas con especialización en Servicio Social a través de una tesis de grado, fue alcanzado solamente por 10 personas, la primera en 1959 y la última en 1980 (UCR, 2013); posteriormente se concreta una Licenciatura en Trabajo Social desde 1965 que también convivió con el certificado de "Trabajador Social" (ucR, 1954), en tanto, algunas personas guardaban derechos para concluir esos tramos formativos.

La cifra de estudiantes en Servicio Social tampoco era elevado, por ejemplo Campos, Molina C., Molina M., Romero y Ruíz (1977), ubican que entre 1942 a 1954 cursaban las materias alrededor de 21 alumnas (os) por año.

De tal manera, puede admirarse la celeridad en que la Escuela de Servicio Social es integrada $y$ paulatinamente, dinamizada para responder a las expectativas académico-universitarias existentes $y$ al proyecto de país que se matizaba y al que se articulaba, desde los años 40 del siglo xx.

Por otra parte, la propia ucR había consignado en su normativa la necesidad de que sus exestudiantes se incorporaran en asociaciones de egresados tal y como se redacta en la cita que sigue:
Artículo 17. La Universidad... Reconocerá y estimulará de igual manera las Asociaciones que formen sus egresados para el mejoramiento de las respectivas profesiones y la realización de otros fines de bien general (Poder Legislativo, 1940).

En el caso de Servicio Social, la vía que se materializó con más presencia fue la de un colegio profesional, institución que tenía un arraigo histórico en la defensa de los intereses gremiales ${ }^{2}$.

Es importante señalar que a pesar de que la Escuela de Servicio Social y los (as) titulados (as) en este campo, tenían una menor presencia en el claustro, sus agentes lograron para el 29 de agosto de $1967^{3}$ que la Asamblea Legislativa aprobara la Ley Orgánica del Colegio de Trabajadores Sociales de Costa Rica (nro. 3943), la cual fue dada a conocer en la corriente legislativa con solo once meses de previo ${ }^{4}$. Dicha norma dictó en su art. 2 que únicamente podían ser parte de ese ente las siguientes personas:

a. Los Licenciados en Servicio Social y en Ciencias Económicas y Sociales, con especialización en Servicio Social de la Universidad de Costa Rica.

b. Los graduados con título de Trabajador Social de la Escuela de Servicio Social de la Universidad de Costa Rica.

$2 \quad$ La influencia y peso político de los Colegios Profesionales en las decisiones de la UCR han sido de tal envergadura, que aún en el siglo Xxi (a junio del 2005) se señala en el artículo 24 del Estatuto Orgánico que una silla del Consejo Universitario está reservada para "un representante designado por la Federación de Colegios Profesionales Universitarios, graduado en la Universidad de Costa Rica".

3 Hasta dos años más tarde, en 1969 se funda el Colegio de Profesionales en Ciencias Económicas y Sociales (Ley nro 4505) donde a pesar de que era posible titularse en esa área con especialidad en Servicio Social, la legislación remitía la incorporación a la organización de trabajadores sociales y no de quienes se egresaban en el campo económico.

4 Los diputados que acogen su presentación fueron Hernán Garrón Salazar, Fernando Volio Jiménez, Graciela Morales Flores y Cecilia González Salazar. 
c. Los graduados en Servicio Social, de universidades extranjeras, cuyos títulos estén reconocidos por la Universidad de Costa Rica.

d. Aquellas personas que tengan certificado de conclusión de estudios en Servicio Social extendido por la Universidad de Costa Rica (Asamblea Legislativa, 1967).

De tal forma esta ley —que es la aún vigente en el país- procuró regular todas las titulaciones existentes en ese momento en el campo del Trabajo Social, además, incluía como fines de esta institución la tarea de promover el progreso de la profesión, velar por que se cumplieran sus principios éticos, defender el interés particular $y$ de grupo de sus colegiados (en ese momento 47 afiliados), colaborar con el Gobierno de la República en las situaciones de emergencia nacional y gestionar o decretar los auxilios que se estimen necesarios para proteger a sus integrantes, entre otros (Torres, 1977).

Por otro lado, es importante acentuar que la situación académica y legal del Trabajo Social da apenas un prisma de lo que se consideraba Servicio Social y asistente o trabajador social en la realidad costarricense, debido a que en el mundo institucional, la cantidad de personas que se habían insertado a laborar en diferentes servicios sociales, sin ningún tipo de preparación especializada o bien, que se rotulaban bajo esas nomenclaturas, era difuso, impreciso y muy amplio.

Cabe aquí la pregunta: ¿Estas personas sin estudios eran trabajadores sociales?, la respuesta se puede desagregar de la siguiente manera:

a) Si para ser trabajador (a) social se requería de un título emitido por la Escuela de Servicio Social (sea certificado de estudios, sea Licenciatura con especialización en Servicio Social, sea Licenciatura en Servicio Social), no lo eran.

b) Si para ejercer el Trabajo Social se requería la tutela del Colegio Profesional que realmente hace una diferencia sobre este aspecto, lo evidente es que desarrollaban un ejercicio de manera ilegal, en tanto lo que dicta la Ley nro 343.
Sin embargo, la historia puede mirarse con otra óptica $y$ hallar en estas mujeres y algunos hombres que laboraban en los servicios sociales, la edificación de una serie de prácticas, acciones, referentes técnicos, instrumentales, operativos y procedimentales valiosos en su momento, donde quizás la formación académica tenía limitaciones o bien, la presión institucional y societal en su conjunto, conllevaban a no desestimar esas contribuciones existentes mucho antes de la fundación de la Escuela antes nombrada.

Gran parte del aporte de estas personas figuraron entre las bases institucionales de las que se nutrió el Trabajo Social costarricense, en mayor o menor medida, según el contexto, la coyuntura, el marco institucional y las demandas que atendía; sin embargo, sus contribuciones no pasarían a ser exclusivamente para estos agentes, sino también para otros gremios como la Enfermería, la Educación Especial y la Orientación Educativa.

Los aportes que esos estamentos de empleados, en su gran mayoría públicos o con estrechos nexos con el Estado no pudieron ser desconocidos por ninguno de los gremios mencionados; un conjunto de estas personas llamadas despectivamente "empíricas" se mostraron en un número considerable de campos profesionales ${ }^{5}$; el Trabajo Social no iba a ser la excepción como se leerá en el siguiente apartado.

\section{RASGOS DE LA REPRODUCCIÓN PROFESIONAL Y LABORAL DEL TRABAJO SOCIAL}

Para 1978, en el mercado de contratación de esta profesión se hallaron 252 personas que se auto declaraban empíricas, 127 entrevistados (as) que alegaban tener algunos estudios

5 La preocupación por el empirismo para quienes se titulaban en cualquier rama universitaria en esos años fue tal, que una de las conclusiones del Primer Congreso Universitario de la UCR en 1946, señaló: e) Celebración de reuniones conjuntas de los integrantes de los Colegios de Egresados, a fin de estudiar los problemas de cada uno de los mismos y pensar en una campaña conjunta de depuración profesional y lucha contra el empirismo (UCR, 1946: 13). Por tanto, no era una cuestión exclusiva que se padeciera únicamente desde el Trabajo Social. 
en este ámbito pero sin ninguna titulación, 60 con formación en otras carreras pero desempeñando cargos en Trabajo Social y 26 que no dieron información al respecto, lo que significaba un total de 465 personas (Altamirano, Araya, Armas, Juárez y Palacios, 1979).

En una línea similar, Guzmán (1973) denunciaba la manera en que las instituciones creaban puestos laborales sin requerimientos académicos, prueba de ello fue la figura de los (as) promotores (as) sociales ${ }^{6}$ (que se asemejaba a un estudiante con tercer año de carrera en las Ciencias Sociales) o también que se contrataba a personas con estudios universitarios, pero provenientes de otros gremios con distintas razones históricas de existencia, tal y como acontecía con las (os) orientadoras (es) educativos.

En ambos casos, subraya la fuente, se hacían estas contrataciones con la intencionalidad de pagar menos salarios, contratar un mayor número de agentes a menor costo $y$ no lidiar con las regulaciones profesionales que establecía la ley de la República.

Sobre los puestos de promotor (a) social el gremio de Trabajo Social ${ }^{7}$ alegaba lo siguiente:

$6 \quad$ Al promotor social en algunos lugares se le denomina como educador social, agente de promoción humana, promotor en salud, promotor en desarrollo de la comunidad o técnico en desarrollo de la comunidad (Colegio de Trabajadores Sociales de Costa Rica, 1979: 14).

7 Entre los rasgos que el Colegio destacaba de estos puestos se señalan: motivación, formación, organización y asesoría de base (comunales, cooperativas, sindicatos, comités de salud, de vivienda, etc.) alrededor de proyectos de salud, vivienda, educación, entre otros, asimismo, el promotor social no podrá desempeñar funciones técnicas con grupos que requieran de tratamiento y terapia. Además, se encargará de la coordinación y administración de actividades específicas correspondientes a proyectos microsociales que se desprenden de las necesidades de los grupos $y / 0$ organizaciones con las que trabaje (por ejemplo, coordinación de actividades con otras instituciones, elaboración de proyectos de trabajo a nivel local o zonal, presupuestos sencillos, etc.). Por otro lado, tendrá a cargo otras funciones: realización de tareas secundarias de investigación, referidas a la elaboración de diagnósticos sobre comunidades u organizaciones de base; ejecutar programas de educación social con grupos, así como, motivar la organización y asesorar la
Debe aclararse que contrario a lo que manifiestan algunas personas desconocedoras del campo del Trabajo Social, la promoción social constituye una función propia del campo de Trabajo Social. Contempla toda la actividad correspondiente a la promoción, organización y capacitación de grupos alrededor de la solución de problemas concretos que los afecta, especialmente a nivel comunal (Colegio de Trabajadores Sociales de Costa Rica, 1979: 14).

Se agrega a lo expuesto, las formas en que la administración pública se configuró bajo parámetros fordistas, tayloristas $y$ weberianos, constituyendo la división técnica del trabajo que se materializó en los servicios sociales y que tuvo sus implicaciones en el campo profesional en estudio. Un ejemplo de esto se localiza en el estudio de Brenes (1974), el cual da cuenta de una complejidad de funciones escalonadas, organizadas y bajo líneas de mando que inicia con personas que trabajan en los servicios sociales con estudios escolares mínimos y culminaba con los cargos más elevados (por ejemplo, llamados puestos de Trabajo Social $4^{8}$ ) que eran ocupados por quienes ostentaban una licencia e incluso, un posgrado en ese campo.

Valga anotar que la pesquisa desarrollada por Torres (1977), también evidenció que las personas con licenciatura en este campo, ingresaban a tareas de alta complejidad y responsabilidad en puestos de mandos medios en diversas instituciones donde se les contrataba (direcciones ejecutivas, administrativas, técnicas y jefaturas de departamentos). Por ejemplo, se da cuenta de su participación en programación, análisis e investigación, así como, proyección presupuestaria en materia de asistencia social

ejecución de proyectos concretos (Colegio de Trabajadores Sociales de Costa Rica, 1979: 14).

8

Considerados como puestos de Dirección general de programas de Desarrollo Social, Bienestar, Seguridad o Promoción Social, con perfiles académicos de maestría o doctorado en Trabajo Social (Colegio de Trabajadores Sociales de Costa Rica, 1979: 13). 
(búsqueda, negociación y coordinación financiera), actividades de asesoría a Presidencias Ejecutivas, Juntas Directivas u órganos colegiados, representación institucional (nacional e internacional), así como, selección y contratación de personal.

Otras jerarquías de menor rango también eran parte del panorama de empleo de estas personas, en especial como jefaturas de oficina (donde se gestaban tareas de orientación, dirección, administración y supervisión) o bien, como asistentes sociales stricto sensu. En ese último escalafón, las tareas estaban asociadas a realizar estudios sociales, elaborar informes, desarrollar intervenciones, ejecutar entrevistas, coordinación interinstitucional, trabajo interdisciplinario $y$ estudios de selección de personal.

El valor de uso de esta categoría, se perfiló por su sesgo interventor en el marco de la vida cotidiana, de la socialización y la sociabilidad que impera en el modo de existir capitalista; aunado a esto, manifiesta un conjunto de direccionalidades ideológicas ( $y$ en bastantes ocasiones doctrinarias) que impregnan sus disimiles praxis anilladas enfáticamente con las lógicas estatales en numerosos complejos que se intercalan con la existencia contextual, coyuntural e histórica del ser social.

Para el caso de la profesión en estudio, el conjunto de personas que laboraban en los servicios sociales era amplio y con un movimiento de fuerza revelador, por ejemplo en el dictamen de la Ley Orgánica del Colegio de Trabajadores Sociales fue evidente lo distintivo que era maniobrar en el mundo del empleo y de la vida institucional de los servicios sociales. Para ejemplificar se puede trascribir un transitorio indicador sobre este aspecto que fue incluido en la legislación señalada:

TRANSITORIO III:

Las personas que a la fecha de la promulgación de esta ley, desempeñen cargos de trabajadores sociales en la administración pública y en las instituciones del estado $y$ quienes hubieren trabajado como tales durante un lapso no menor de cinco años podrán continuar trabajando bajo esa denominación y participar en los concursos para puestos que requieran conocimientos en Servicio Social, sin perjuicio del artículo 12 de la presente Ley.

El acotamiento anterior cobra todavía más sentido al localizarse que desde el 14 de agosto de 1953, se había constituido la Asociación Nacional de Trabajadores Sociales (anTs), órgano que aglutinaba un diverso grupo de 85 agentes que se reconocían con vínculos a esta profesión o bien, a los servicios sociales, en especial se destacaban asalariadas (os) del Patronato Nacional de la Infancia $(1930)^{9}$ (Torres, 1977).

Las luchas de ese colectivo gremial orbitaban en el tema de los bajos salarios, las condiciones de trabajo, el reconocimiento estatal y los roces con personas con estudios en otras áreas como Educación y Enfermería (con quienes generalmente se había mantenido una relación histórica en muchas aristas, consúltese Mora, 1968).

Quienes integraban la AnTs forjaron gran parte de las presiones para que se fundara el Colegio de Trabajadores Sociales; dicha asociación paulatinamente fue perdiendo vigencia en razón de que para 1969, modificó sus estatutos y con ello permitió únicamente el ingreso a sus filas a graduadas (os) en el campo del Servicio Social, excluyendo a otras figuras como asistentes o promotores sociales que exclusivamente tenían significado en las esferas institucionales (Grupo Yurustí, 1982).

Torres (1972) subraya que para 1971, el número de personas con licenciatura relacionada con el Servicio Social o el Trabajo Social eran solamente 65 (un 83\% mujeres), ubicadas en 22 instituciones del Estado; se estimaba que

$9 \quad$ Entidad donde según Casas (1975), se usaba primigeniamente el término de "visitadoras sociales"; por el contrario, García y Zamora (1982) asienten que esa referencia se inaugura en la Clínica Infantil adscrita a la Secretaría de Gobernación y Policía (1920). Sin embargo, Araya, Calderón, Chinchilla y Vega (1986) afirman que es en la Secretaría de Salud Pública y Protección Social (1927) donde se refiere por primera vez al uso de un vocablo vinculante, esta vez de asistentes sociales. 
era un profesional en esta área por cada 22000 habitantes.

Relacionado con lo anterior, Jiménez (1968) afirmaba que en esas décadas, la mayoría de los (as) profesionales se ubicaron en el Estado, específicamente en organismos del gobierno central y sus instituciones de carácter autónomo y semiautónomo, algunos eran subvencionados total o en parte por el erario público $y$ otros, con fondos especiales.

Los campos de inserción laboral para 1978, se concentraban en el sector salud (36\%), justicia (26\%), bienestar social (15\%), promoción social (13\%) y educación (8\%) (Mora, Murillo, Oviedo y Segura, 1978). En esta década el sector privado de contratación era prácticamente nulo (Altamirano et ál., 1979).

El tema salarial es otra de las discutas que generalmente aparecen en las aprehensiones de la categoría profesional, al respecto cabe afirmar que para quienes ostentaban certificaciones de licenciatura se denotaba una condición favorable en comparación con otros agentes en similares niveles académicos dentro de la administración pública ${ }^{10}$. No obstante, fue posible distinguir diferencias en una desigualdad que afectaba a los y las profesionales en estudio si se les comparaba con médicos, abogados, economistas, odontólogos, farmacéuticos e ingenieros.

Esta preocupación que movilizaba y organizaba un número importante de personas relacionadas con los servicios sociales, se hacía sentir desde mitad del siglo xx (Grupo Yurustí, 1982); en los años 60, la Escuela de Servicio Social realizó un estudio que señaló una serie de consideraciones en materia salarial y de condicionantes laborales, el cual fue acogido por algunas instituciones del Estado (Mora, 1968). Sobre este tema, una de las fuentes identificadas reseña:

No es un misterio los bajos ingresos económicos que percibían los servidores sociales hace algunos años y aunque en la actualidad continúan siendo bajos para

10 Los hombres titulados en esta rama evidencian un mayor salario desde la década de los 70 del siglo xx (Torres, 1972). una proporción de estos profesionales, se equipara con el de otros graduados universitarios que tradicionalmente han recibido un monto de ingresos, como remuneración a su trabajo acorde a su nivel profesional (Torres, 1972: 18).

En una línea similar se subraya:

...la remuneración salarial del Trabajador Social no es baja con relación a la de otros profesionales... De considerable importancia es el caso de instituciones que cuentan con su propio escalafón de salarios (pues en algunas de ellas este hecho resulta favorable para el profesional) tales como el Instituto Nacional de Seguros, Instituto Nacional de Vivienda y Urbanismo y la Corte Suprema de Justicia, debido a que remuneran adecuadamente al Trabajador Social. Ocurre lo contrario en instituciones como la Caja Costarricense del Seguro Social, Patronato Nacional de la Infancia e Instituto Mixto de Ayuda Social, en donde el escalafón existente no favorece al Trabajador Social. Cabe anotar que estas últimas instituciones absorben un número considerable de profesionales en esta disciplina (Altamirano et ál., 1979: 105-113).

Otro dato revelador, siguiendo el anterior estudio, es que para 1979, se sumaban 149 licenciadas (os) en esta rama, 98 personas egresadas (pendientes de defender su trabajo final de graduación para licenciarse), 56 bachilleres en Trabajo Social (título de pre-grado que se consolida en la década de los 70) y 12 asistentes o trabajadores (as) sociales con certificados de la Escuela respectiva, para un total de 320 personas con estudios propios en el campo.

Sumado a esto, los años 70 reflejaron el impulso para que profesionales en este campo se consolidaran en las últimas dos décadas del siglo xx en empresas estatales (Refinadora Costarricense de Petróleo-REcope, Instituto Costarricense de Electricidad-ice e Instituto Nacional de Seguros-ins) y en mucho menor medida, se trataran de filtrar en el campo empresarial privado 
(Demasa-Jugos del Campo y Kimberly Klark) o público no estatal como cooperativas (cEcoop, Coopeseice RL, Cooperativa de Productores de Leche Dos Pinos) (Araya y Castillo, 1996).

En ese mismo tiempo, el Colegio Profesional emitió un manual descriptivo de funciones, el cual caracterizó la labor de estos agentes. A continuación se presentan las funciones típicas y de carácter genérico del ejercicio del trabajo social en Costa Rica en 1979:

1) Estudiar, diagnosticar y definir la acción social (intervención primaria, secundaria $y$ terciaria) correspondiente con individuos, grupos, comunidades y organizaciones sociales.

2) Dirigir el diseño y planeamiento de investigaciones sociales $y$ la ejecución $y$ análisis de los resultados de las mismas.

3) Planear, dirigir, supervisar y evaluar programas $y$ proyectos variados de seguridad, bienestar y promoción social.

4) Supervisar el trabajo del personal asignado a los programas y proyectos de bienestar, seguridad y promoción social.

5) Establecer mecanismos de coordinación y velar porque se ejecuten con otras dependencias o instituciones que tengan relación con los programas y proyectos a su cargo.

6) Coordinar con otros profesionales del equipo de trabajo en que labore.

7) Establecer pautas de supervisión para unidades de trabajo o grupos de profesionales y/o técnicos que laboren en funciones propias del Trabajo Social o afines.

8) Establecer pautas de supervisión para unidades de trabajo o grupos de profesionales y/o técnicos destacados en programas de bienestar, seguridad y promoción social.

9) Administrar, dirigir y supervisar programas y proyectos de desarrollo social (en los campos del bienestar, seguridad o promoción social).

10) Asesorar el planeamiento, ejecución y evaluación de programas y proyectos sociales de instituciones públicas, empresas privadas y organismos sociales privados.
11) Capacitar a voluntarios, grupos, organizaciones y líderes que trabajan en programas de bienestar, seguridad y promoción social (programas y proyectos de desarrollo social).

Para inicios de los años 80 del siglo xx, escenario de penetración neoliberal y de controversias en el campo de la protección y la seguridad social (Molina y Sáenz, 1994), el campo laboral de la profesión se confrontó con debates sobre la contratación, los regímenes salariales, la materialización de los servicios sociales, las recalificaciones $y$ estrategias de reforma de las instituciones donde el Trabajo Social había legitimado su existencia en Costa Rica, léase sobre este particular:

... durante los últimos diez años se ha observado que profesionales en otras disciplinas han venido usurpando campos de acción propios de nuestra profesión... en algunas instituciones se ha llegado a eliminar las oficinas de Trabajo Social y su Jefatura... en algunos centros laborales son profesionales de otras disciplinas quienes dirigen $y$ supervisan las funciones de colegas de Trabajo Social (Colegio de Trabajadores Sociales, 1981: 23).

En este marco histórico se localizó una tendencia significativa para perfilar a la profesión en tareas de promoción, asistencia y administración de programas sociales, situándose a su vez un ocaso en tareas de investigación e incidencia en formulación de políticas institucionales clave (Mora, Morera y Ramírez, 1992).

El tema del desempleo profesional fue también destacado en un estudio con datos de 1986, el caso de Trabajo Social (entrevistando a 55 licenciadas y 28 bachilleres) proyectó un $12,5 \%$ en dicho campo. Entre las razones de ese cese se localizaban problemas de salud, deseos de no laborar, pero también algunas dificultades de encontrar opciones de contrato (Consejo Nacional de Rectores-conare, 1989).

Según un perfil profesional desarrollado en 1994, los rasgos del trabajo profesional se componían por una tendencia marcada en la asistencia social $(31,26 \%)$, la investigación 
microsocial $(18,75 \%)$, la administración, el tratamiento terapéutico junto a la promoción social $(12,6 \%)$ y finalmente, la educación y la prevención $(6,25 \%)$ (Colegio Trabajadores Sociales, 1994).

En esas mismas décadas, se materializaron algunas expresiones muy limitadas de lo que se denomina "ejercicio liberal", el cual se caracterizaba por cobro de honorarios sin ser parte de la planilla de la organización. Entre los servicios que se ofertaban se localizaron las asesorías sociales, consultas familiares o individuales, investigaciones, evaluaciones y elaboración de estudios especializados (tales como diagnósticos, pericias, valoraciones, entre otros) (Aguilar, Araya y Murillo, 1990).

Otra referencia de contratación para estos tiempos se ubica en el campo del tercer sector, acompañando un crecimiento importante de organizaciones no estatales o entes privados que proveen servicios al Estado $\mathrm{u}$ organismos internacionales en el contexto de consolidación de las orientaciones neoliberales (Picado, 2002).

De tal manera, la situación de reproducción laboral del Trabajo Social en Costa Rica fue tensada durante los años en estudio por varios ejes, los cuales tuvieron un arraigo estrictamente en las relaciones sociales y sus alteraciones en la vida que se entreteje en este país.

\section{CONCLUSIONES}

El Trabajo Social se consolidó en Costa Rica por tres vías relevantes: a) adquiere una base de certificación académico-universitaria de su especialidad exclusivamente; b) gesta una organización gremial avalada, sancionada, reconocida y estimulada por el Estado y las fuerzas políticas hegemónicas; c) tiene a su haber un mercado de trabajo y contratación diverso $y$ de difícil delimitación, pero que en ningún momento le dificulta su inserción en cargos asalariados.

Analizar desde atrás el campo del Trabajo Social y su desarrollo internacional, en América Latina y Costa Rica, brinda una panorámica entramada por permanentes luchas de consolidación y defensa de ese cuerpo profesional, tanto en su existir académico como laboral.
La historia costarricense le posibilitó al Trabajo Social, un particular marco institucional de empleabilidad y académico que signaron su génesis y desenvolvimiento.

En lo que respecta al espectro académico $y$ en relación con lo acontecido en otras latitudes, la institucionalidad universitaria incorpora en forma expedita $y$ con realce, a la primera Escuela de Servicio Social.

La ucr no escatimó en razón de los intereses por los que se funda, para darle soporte académico e intelectual a la formación, asî como acompañar la búsqueda de legitimidad en la certificación de los estudios en este campo.

Por su lado, el Poder Legislativo también brindó un apoyo significativo a sus titulados para que por medio de la legalización respectiva se controlara, sancionara y reconociera el ejercicio de dicha profesión.

La vida institucional y sus diversas prácticas asociadas a los servicios sociales, dieron una interesante pero dificultosa plataforma de acciones, agentes y pautas para el desenvolvimiento de los $y$ las profesionales en Trabajo Social que difuminaba $y$ atenuaba sus rasgos, trayectoria y méritos académicos ante una compleja gama de actores y quehaceres, que en lo inmediato, semejaban prácticas y atribuciones ante los ojos cotidianos de la ciudadanía, en relación con los campos de empleo de estas (os) agentes.

Otros conjuntos profesionales también se traslaparían con las acciones existentes en las instituciones relacionadas con la política social. En el caso del Trabajo Social, este se arraigó como una pieza más, en un conjunto de intelectuales requeridos para la intervención estatal en la malla de presiones existentes ante la "cuestión social".

A pesar de existir un marco legal y reglamentario que ha subsidiado la práctica del Trabajo Social, las tensiones entre el gremio y el propio Estado denotaron ser permanentes y plurales, en tanto se expresaron acciones institucionales que condujeron a la creación de puestos con contenidos que burocráticamente, minimizaban el requerimiento de pericia académica calificada, como acontece con la llegada de la figura de los (as) promotores sociales. 
Por su lado, se encuentra una gama de campos de trabajo con salarios, demandas $y$ reconocimientos diferenciados, los cuales gestaron fracturas a lo interno del gremio sobre su agenda de logros y reivindicaciones, astillando desde luego los márgenes de integración.

Las posibilidades de ampliar el mercado de inserción profesional en estos años fueron dificultosas en ámbitos como la empresa privada, la industria y el ejercicio liberal, un margen también estrecho fue ubicable en las empresas públicas, cooperativas y algunas organizaciones no estatales.

Según la búsqueda bibliográfica desarrollada, el desempleo también tuvo su presencia en este colectivo gremial tal y como lo evidenciaron las fuentes anotadas, aunque este último aspecto ha sido poco indagado y problematizado para dicha profesión.

Finalmente, cabe anotar que al escribirse este artículo a más de una década del siglo xxi, el ejercicio de recorrer esta trayectoria histórica vigoriza la comprensión densa y compleja que significa descifrar las condiciones en que el Trabajo Social se ha anillado en la sociedad costarricense y los complejos que definen su existir.

\section{BIBLIOGRAFÍA}

LIBROS

Acuña, Víctor y Molina, Iván. Historia económica y social de Costa Rica (17501950). 1era. edición. San José, Costa Rica: Editorial Porvenir, 1991.

Alayón, Norberto. Hacia la historia del Trabajo Social en la Argentina. 1era. edición. Lima, Perú: Centro Latinoamericano de Trabajo Social-cELATs, 1980.

Beeche, Héctor. Servicio social criminológico. 1era. edición. Habana, Cuba: Editorial Jesús Montero, 1951.

Calderón, Manuel. "Proceso y estructura del liberalismo en Costa Rica (1812-1882)". Desarrollo institucional de Costa Rica: de las sociedades indígenas a las crisis del 30. 1era. Edición. Jaime Murillo (comp.). San José, Costa Rica. Editorial Guayacán, 1988: 23-35.
Consejo Nacional de Rectores-Conare. La situación laboral y otras características de los graduados de 1986 de las universidades estatales. 1era. Edición. San José, Costa Rica: Oficina de la Planificación de la Educación Superior, 1989.

De la Cruz, Vladimir. "La educación y la cultura costarricense en el siglo XIX: de las Cortes de Cádiz a las reformas educativas". Historia de la educación costarricense. lera. edición. Jorge Salazar (comp.). San José, Costa Rica. Editorial de la Universidad Estatal a Distancia-Euned y Editorial de la Universidad de Costa Rica-EUCR, 2003: 33-42.

Esquivel, Freddy. Trabajo Social en Costa Rica: del ideario liberal a su constitución en el reformismo. 1era. edición. San José, Costa Rica: Editorial de la Universidad de Costa Rica-EUCR, 2007.

González, Yamileth. "La Universidad de Santo Tomás: una polémica sin cuartel. Centro de Investigaciones Históricas". Historia de la Educación Superior en Costa Rica. Universidad de Costa Rica. 1era. Edición. San José, Costa Rica: Editorial de la Universidad de Costa Rica-EUCR. 1991: 17-27.

González, Yamileth. "Educación diversificada y humanista para una democracia integral (1950-1970)". Historia de la educación costarricense. 1era. edición. Jorge Mario Salazar (comp.). San José, Costa Rica: Editorial de la Universidad Estatal a Distancia-EunED y Editorial de la Universidad de Costa Rica-EUCR, 2003: 30-42.

Manrique, Manuel. De apóstoles a agentes de cambio. lera. edición. Lima; Perú: Centro Latinoamericano de Trabajo Social-CELATS, 1982.

Martinelli, María. Servicio Social: Identidad y Alienación. 1era. edición en castellano. São Paulo, Brasil: Editora Cortez: 1997.

Molina, Iván y Palmer, Steven. Educando a Costa Rica. Alfabetización popular, formación docente y género (1880- 
1950). 1era. edición. San José, Costa Rica: Editorial de la Universidad Estatal a Distancia-EUnED, 2003.

Netto, José. Capitalismo monopolista y Servicio Social. 1era. edición en castellano. São Paulo, Brasil: Editora Cortez, 1992.

Obregón, Rafael. Hechos militares y políticos. 2 da. Edición. Alajuela, Costa Rica: Museo Histórico Cultural Juan Santamaría, 1981.

Quesada, Juan. Un siglo de educación costarricense 1814-1914. 1era. edición. San José, Costa Rica: Editorial de la Universidad de Costa Rica-EUCR, 2005.

Tinoco, Luis. La Universidad de Costa Rica. Trayectoria de su creación. lera. edición. San José, Costa Rica: Editorial de la Universidad de Costa Rica-EucR, 1983.

Yglesias, Enrique. La Escuela de Ciencias Económicas y Sociales. Estudio sobre su desarrollo y organización académica. lera. edición. San José, Costa Rica: Editorial Universitaria, 1953.

\section{PUBLICACIONES PERIÓDICAS}

Castillo, Raúl. "La formación profesional de trabajadores sociales en América Latina". Revista Acción Crítica 8. Lima, Perú. Centro Latinoamericano de Trabajo Social, diciembre 1980: 40-52.

Esquivel, Freddy. "Neoliberalismo en Costa Rica: secuelas en la cuestión social durante el siglo XX". Revista Cátedra Paralela 9. Rosario, Argentina. Escuela de Trabajo Social de la Universidad del Rosario, marzo 2012: 76-101.

International Association Schools of Social Work (IASSW). "Report Census". Revista Social Dialogue 4. Enero 2013: 52-55.

Lima, Leila. "Desarrollo del Trabajo Social en América Latina". Revista Acción Crítica 8. Lima, Perú. Centro Latinoamericano de Trabajo Social, junio 1980: 29-40.

Molina, Lorena y Sáenz, Jorge. "Trabajo Social: lo académico y lo profesional". Revista de Ciencias Sociales 64. San José, Costa Rica. Universidad de Costa Rica, junio 1994: 101-107.

Mora, Marilú; Morera, Nidia y Ramírez, María. "El perfil profesional del trabajador social". Revista de Ciencias Sociales 56. San José, Costa Rica. Universidad de Costa Rica, junio 1992: 15-21.

Picado, Marta. "Las ONG: escenario para una intervención fundada de las y los trabajadores sociales". Revista Costarricense de Trabajo Social 14. San José, Costa Rica. Colegio de Trabajadores Sociales de Costa Rica, julio 2002: 6-12.

\section{TESIS NO PUBLICADAS}

Aguilar, Florizul; Araya, Juanita y Murillo, Elisa. "El Trabajo Social liberal en la prestación de servicios profesionales en Costa Rica". [Memoria de Seminario de Licenciatura en Trabajo Social]. Universidad de Costa Rica, 1990.

Altamirano, Trilce; Araya, Aura; Armas, Jeannete; Juárez, Zeneyda y Palacios, Mireya. "El desajuste entre la concepción $y$ las actividades de la profesión de Trabajo Social en Costa Rica". [Memoria de Seminario de Licenciatura en Trabajo Social]. Universidad de Costa Rica, 1979.

Araya, Lorena y Castillo Walter. "Características generales del Trabajo Social de empresa pública y privada". [Tesis de Licenciatura en Trabajo Social]. Universidad de Costa Rica, 1996.

Brenes, Adelina. "La consulta externa y la función del Trabajador Social en las clínicas periféricas de la Caja Costarricense de Seguro Social". [Tesis de Licenciatura en Trabajo Social]. Universidad de Costa Rica, 1974.

Campos Morera, Ivette; Molina Cruz, Emilia; Molina Molina, María; Romero Rodríguez, Carmen y Ruíz Rojas, Ana. "Evolución social de la estructura académica de la Escuela de Trabajo Social en Costa Rica". [Tesis de Licenciatura en Trabajo Social]. Universidad de Costa Rica, 1977.

Casas, Gerardo. "Evolución histórica del Servicio Social costarricense". [Tesis de Licenciatura en Trabajo Social]. Universidad de Costa Rica, 1975.

García, Esmeralda y Zamora, Mireya. "Evolución del Bienestar en la Dirección 
de Bienestar Social del Ministerio de Trabajo y el Sub Programa de Asistencia Social en el IMAS". [Tesis de Licenciatura en Trabajo Social]. Universidad de Costa Rica, 1982.

Guzmán, Laura. "Una estrategia para el cambio en el Trabajo Social Costarricense $y$ de la sociedad: aspectos tácticos, metodológicos y estructurales". [Tesis de Licenciatura en Trabajo Social]. Universidad de Costa Rica, 1973.

Mora, Rosa. "Algunos aspectos relacionados con la formación de profesionales en la Escuela de Servicio Social de la Universidad de Costa Rica". [Tesis de Licenciatura en Ciencias Económicas y Sociales con especialización en Servicio Social]. Universidad de Costa Rica, 1968.

Mora, Virgilio; Murillo, Dinorah; Oviedo, María Rosa y Segura, Zaida. "Análisis de los factores socioculturales que contribuyen en la elección de la carrera de Trabajo Social en la sede central y centros regionales". [Tesis de Licenciatura en Trabajo Sociall. Universidad de Costa Rica, 1978.

Torres, Víctor. "Las organizaciones profesionales y gremiales del Trabajo Social en Costa Rica en relación con las limitaciones, la Escuela de Trabajo Social y la Reconceptualización del Servicio Social". [Tesis de Licenciatura en Trabajo Social]. Universidad de Costa Rica, 1977.

\section{TEXTOS ELECTRÓNICOS}

Escuela de Trabajo Social. Directorio Latinoamericano de Trabajo Social, 2013. En: <http://www.ts.ucr.ac.cr/ directorio.htm $>$ [consultado el 12 de mayo de 2013].

International Federation of Social Worker. What we do, 2013. En: <http://ifsw.org/ what-we-do/> [consultado el 5 de mayo de 2013].

OTROS

Acosta, Martha; Bohórquez, Myriam; Duarte, Flor; González, Adriana y Rodríguez,
Clara. (2012). Trabajo Social en el área de la salud. Perfiles y competencias profesionales básicas en el área de la salud. Bogotá, Colombia.

Araya, Rosa; Calderón, Manuel; Chinchilla, José y Vega, Cecilia. Análisis del surgimiento y desarrollo del Servicio Social en Costa Rica y su ubicación en el contexto económico, social y político. Informe final de investigación. Alajuela, Costa Rica: Universidad de Costa Rica, 1986.

Asamblea Legislativa de la República de Costa Rica. "Ley nro. 3643 Ley Orgánica del Colegio de Trabajadores Sociales de Costa Rica”. San José, Costa Rica. 29 de agosto de 1967.

Colegio de Trabajadores Sociales de Costa Rica. Manual descriptivo de funciones. San José, Costa Rica, 1979.

Colegio de Trabajadores Sociales de Costa Rica. Seminario de diagnóstico de Trabajo Social en las instituciones. San José, Costa Rica, 1981.

Colegio de Trabajadores Sociales de Costa Rica. Perfil profesional del quehacer del Trabajo Social. San José, Costa Rica, 1994.

Congreso constitucional. Decreto Nro LXXVII. Decreto de clausura de la Universidad de Santo Tomas. Costa Rica, 1888.

Conselho Federal de Serviço Social (CFEss). Sobre a Incompatibilidades entre gradua ão à distancia e Serviço Social. Brasilia, Brasil, 2010.

Grupo Yurustí. Documento conmemorativo del $x v$ aniversario de la creación del Colegio de Trabajadores Sociales de Costa Rica. San José, Costa Rica: Colegio de Trabajadores Sociales de Costa Rica, 1982.

Jiménez, Victoria. Manual descriptivo de funciones y sistemas de referencia en las instituciones de bienestar social y servicios sociales establecidos en Costa Rica. San José, Costa Rica: Ministerio de Trabajo y Bienestar Social, 1968.

Poder Legislativo de la república de Costa Rica. "Ley nro. 362 Ley orgánica de la Universidad de Costa Rica". La Gaceta 101. San José, Costa Rica, 1940. 
Torres, Oscar. Un estudio de utilización de los profesionales en Servicio Social y el mercado de trabajo. San José, Costa Rica: Oficina de Planificación Universitaria. Universidad de Costa Rica, 1972.

Universidad de Costa Rica. Conclusiones del Primer Congreso Universitario. San José, Costa Rica: Universidad de Costa Rica, 1946.

Universidad de Costa Rica. Escuela de Ciencias Económicas y Sociales. San José, Costa Rica: Imprenta Trejos Hermanos, 1948.

Universidad de Costa Rica. Prospecto del Departamento de Sociología y Servicio
Social. San José, Costa Rica: Imprenta Trejos Hermanos, 1954.

Universidad de Costa Rica. Guía para los estudiantes. Curso Lectivo 1955. San José, Costa Rica: Imprenta Trejos Hermanos, 1955.

Universidad de Costa Rica. Datos de graduados Facultad de Ciencias Económicas y Sociales. Setenta aniversario. San José, Costa Rica, 2013.

Fecha de ingreso: 12/03/2013 Fecha de aprobación: 30/01/2014 